\title{
Target selection for reaching and saccades share a similar behavioral reference frame in the macaque
}

\author{
Hansjörg Scherberger ${ }^{1}$, Melvyn A. Goodale ${ }^{2}$, \\ and Richard A. Andersen ${ }^{1}$
}

${ }^{1}$ Division of Biology, California Institute of Technology, Pasadena, CA 91125.

${ }^{2}$ Department of Psychology, University of Western Ontario, London, Ontario N6A5C2.

\section{Correspondence should be sent to:}

Richard A. Andersen

Division of Biology

California Institute of Technology

Pasadena, CA 91125

Tel: (626) 395-8336

andersen@vis.caltech.edu

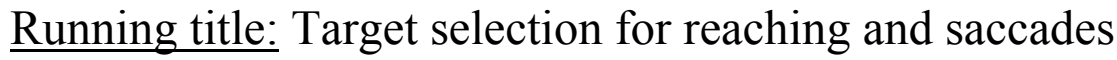

Number of words in the abstract: $\quad 249$

Number of text pages: $\quad 34$

Number of figures: $\quad 12$

Number of tables: $\quad 2$

Accepted for publication in the Journal of Neurophysiology

November 18, 2002 


\begin{abstract}
The selection of one of two visual stimuli as a target for a motor action may depend on external as well as internal variables. We examined whether the preference to select a leftward or rightward target depends on the action that is performed (eye or arm movement) and to what extent the choice is influenced by the target location. Two targets were presented at the same distance to the left and right of a fixation position and the stimulus onset asynchrony (SOA) was adjusted until both targets were selected equally often. This balanced SOA time is then a quantitative measure of selection preference. In two macaque monkeys tested, we found the balanced SOA shifted to the left side for left-arm movements and to the right side for right-arm movements. Target selection strongly depended on the horizontal target location. By varying eye, head, and trunk position, we found this dependency embedded in a head-centered behavioral reference frame for saccade targets and, somewhat counter-intuitively, for reach targets as well. Target selection for reach movements was influenced by the eye position, while saccade target selection was unaffected by the arm position. These findings suggest that the neural processes underlying target selection for a reaching movement are to a large extent independent of the coordinate frame ultimately used to make the limb movement, but are instead closely linked to the coordinate frame used to plan a saccade to that target. This similarity may be indicative of a common spatial framework for hand-eye coordination.
\end{abstract}




\section{Introduction}

In everyday life, we most often look at a target before we reach to it. In fact, it is more difficult to reach out to a target while maintaining fixation elsewhere, whereas the opposite, namely looking at a new target while keeping the arm at another location, is trivially easy (Land et al., 1999). Many have argued that the planning of saccades and shifts of attention are closely linked and share much of the same neural circuitry (Rizzolatti et al., 1987; Deubel and Schneider, 1996; Corbetta et al., 1998). In fact, a saccadic eye movement to a target could be considered a physical instantiation of a shift of attention. Also the eyes look to reach locations to bring the fovea on the target, presumably to increase the accuracy of the final stage of the hand path (Ballard et al., 1995; Johansson et al., 2001).

Bearing in mind these considerations, one might predict that the process of selecting targets for reach movements would use the same frame of reference as the selection of targets for saccades - even though from a motor planning perspective the mechanics for arm movements and eye movements are quite different (Gielen et al., 1984; Frens and Erkelens, 1991; McIntyre et al., 1997). Because eye and hand movements are tightly linked, moving the eyes away while reaching is not easy (Neggers and Bekkering, 2000; Johansson et al., 2001). Since eye movements and shifts of attention occur much more frequently than hand movements, the frame of reference that is used for the planning of eye movements may dominate in any target selection process, including the selection of targets for arm movements.

What is the frame of reference in which saccade targets are selected? Eye movements are constrained within the orbit by the oculomotor mechanics (Ruete, 1855; Robinson, 1975). All other decision variables being equal, saccade targets might therefore be preferred that bring the eye back to the head-centered midline (Yarbus, 1967; Carpenter, 1988; Desmurget et al., 1998). In other words, saccade target selection under these conditions would occur in a head-centered reference frame. One would also expect that saccade targets that are closer to the foveal center on the retina are preferred as well, since these targets are represented in the visual system at a higher resolution (Weymouth, 1958; Ballard et al., 1992), which would predict a co-existing retinocentered frame of reference for saccade target selection. 
Alternatively, it could be argued that target selection for a reaching movement is dominated by the mechanics of arm movements (Soechting and Flanders, 1992; Flanders and Soechting, 1995). If this were the case, the spatial reference for target selection would differ for arm and eye movements and reach selection would be embedded within a trunk-centered reference frame.

Apart from more abstract frames of reference, there may also be a spatial bias for targets to the left or right depending upon which arm is reaching (Fisk and Goodale, 1985). In other words, there could be a laterality effect for arm movements overlaying a basic head- or trunk-centered reference frame.

We measured the preference to select targets for saccade and reach movements in two behaving monkeys. We presented two visual targets equidistantly on either side of a fixation position (FP) and adjusted the stimulus onset asynchrony (SOA) of the targets (with the nonpreferred target presented first) until the animal selected both targets equally often. The balanced SOA time was then taken as a quantitative measure for the preference for target selection, and we systematically varied the position of the eye, head, and trunk along the horizontal axis to determine the frame of reference used for these decisions.

We found that target selection for left-arm movements was shifted to the left side and for right-arm movements shifted to the right side. Nevertheless, target selection for left- and rightarm movements as well as saccades was dependent on the target positions, and this dependency was embedded in a head-centered reference frame for both saccades and reaches.

Part of this study has been published in abstract form (Scherberger et al., 1999). 


\section{Methods}

\section{Subjects}

Two male rhesus monkeys (Macacca mulatta) participated in this study. To prepare for the behavioral experiments, two surgical procedures were performed in both animals under sterile conditions and general anesthesia (pentobarbital sodium $10 \mathrm{mg} / \mathrm{kg}$ intravenously or isofluorane 1$2 \%)$. Heart rate, respiration rate, and body temperature were continuously monitored throughout each procedure. First, a custom-made stainless steel or titanium head post and a dental acrylic head cap (Coralite Duz-All ${ }^{\mathrm{TM}}$ ) were implanted onto the skull of each animal. In a second procedure, a scleral search coil was then implanted in one eye to monitor the animal's eye position (Judge et al., 1980). Systemic antibiotics and analgetics were administered for several days after each surgery, and animals were allowed to recover for at least one week before behavioral training began.

All surgical and animal care procedures were in accordance with the National Institute of Health guidelines and were approved by the California Institute of Technology Institutional Animal Care and Use Committee.

\section{Figure 1 about here}

\section{Setup}

The monkeys were seated upright in individually adjustable primate chairs and their trunk was fixed to the back rest of the chair using Velcro Strips ${ }^{\mathrm{TM}}$. One arm was immobilized using a restraining band at the animal's elbow. The head was fixed to the chair using a head holder apparatus that connected to the animal's head post. Head fixation position could be rotated along an earth-vertical axis that went through the center of the head. The position of the chair, and hence the trunk, could also be varied along the same vertical axis as the head by means of a motorized turntable. For clarity, head and trunk positions are always expressed with respect to the room (space coordinates) in this paper. 
A cylindrical-shaped reach board (surface radius $26 \mathrm{~cm}$ ) was positioned in front of the animal such that the axis of the cylinder coincided with the rotation axis of the head and trunk. An array of pushbuttons (three horizontal rows of nine buttons; spacing of $16^{\circ}$ visual angle) was mounted on the board with the center button located at straight-ahead to the animal. Each pushbutton (diameter $3.7 \mathrm{~cm}$ ) contained a red and a green LED that were located at its center behind a $1.2 \mathrm{~cm}$ translucent lens.

After recovery from surgery, animals were trained, in otherwise total darkness, to visually fixate red LED lights and to reach out and touch buttons that were illuminated green. During training and experiments, horizontal and vertical eye positions were recorded with a sampling rate of $250 \mathrm{~Hz}$, while the event times of LED illumination and button-press and -release were recorded with a 2-ms precision.

\section{Experimental protocol}

All trials began with monkeys fixating (within a window of $\pm 2.7^{\circ}$ ) and touching a red and green illuminated button, which we refer to as the fixation position (FP). Then, after a variable delay of 500-1000 ms, either one or two targets were illuminated, while at the same time the lights at the FP were extinguished.

In single reach trials, a target button located $16^{\circ}$ to the left or the right of the FP was illuminated green, and both LEDs at the FP were turned off. The monkey was required to release the FP button and reach to the target button while maintaining eye fixation at the FP. In single saccade trials, a left or a right target was illuminated in red, and the animal was required to make an eye movement to fixate the target LED while continuing to press the FP button.

In double stimulation trials, a second target of the same color was presented in the opposite direction of the first target at a distance of $16^{\circ}$ from the FP (Fig. 1A). The second target was presented either simultaneously or with a variable time delay with respect to the first target, and the animal was free to choose one of the two visual stimuli as the movement goal.

We refer to the time delay between the first and the second target as the stimulus onset asynchrony (SOA), which was altered during the trial sequence of each run using an adaptive staircase procedure (PEST: parameter estimation using sequential testing). In this adaptive 
procedure, the less preferred target was presented earlier than the preferred one, which increased its frequency of selection, and this time lead was adjusted such that both targets were selected equally often (Taylor and Creelman, 1967; for reviews see Macmillan and Creelman, 1991; Gescheider, 1997). This time we call the balanced time delay (BTD).

Single and double stimulation trials were considered successful, when the monkey acquired only one target by performing the required action. When this occurred the monkey was rewarded with a drop of juice. The amount of reward was independent of the animal's choice and was held constant during each run (Platt and Glimcher, 1997).

A run was defined as a sequence of single and double stimulation trials of the same type (left or right arm reaches or saccades) that were presented randomly interleaved at 3-4 different horizontal FPs, while the head and trunk position was kept constant (Fig 1B). At each FP, single trials to the left and to the right were interleaved with double stimulation trials (ratio: 1:1:2) for a total of 80-100 trials (Fig.1C). The SOA in the double stimulation trials was thereby altered by the adaptive procedure separately for each FP.

On each experimental day, we tested one of the conditions: left-arm reaches, right-arm reaches, and saccades by varying either head position or trunk position in a series of runs. The sequence of positions tested was alternated between $\left(0^{\circ}, 16^{\circ},-16^{\circ},-8^{\circ}, 8^{\circ}, 0^{\circ}\right)$ and $\left(0^{\circ},-16^{\circ}, 16^{\circ}\right.$, $\left.8^{\circ},-8^{\circ}, 0^{\circ}\right)$ between experimental days. To account for measurement variation, all conditions were repeated at least three times on different experimental days in each animal.

\section{Data analysis}

We defined the response time (RT) as the time between the presentation of the first target and the time when a target was acquired. Further, we defined as the movement time (MT) the time between the release of the FP button and press of the target button in the case of reach movements, and the saccade duration (time period with eye velocity exceeding $50 \%$ s) for eye movements.

In an off-line analysis, we determined the BTD of the SOA using all trials of each condition by modeling the relationship between target selection preference and SOA in a psychometric function fit using the logistic distribution 


$$
P(S O A, \alpha, \beta)=1 /\left(1+\exp \left(\frac{\alpha-S O A}{\beta}\right)\right),
$$

where $P$ is the probability for a rightward choice at a given SOA. Assuming a binomial distribution (that is, the probability for a leftward choice is 1-P). We fitted the parameters $\alpha$ and $\beta$ by determining the maximum likelihood of the joint distribution of all trials for the given data set (Treutwein, 1995) and then defined BTD as $\alpha$, which is the SOA for which the probabilities of leftward and rightward choices are 0.5 .

In order to compare changes of BTD for different FPs and to calculate the shifts of response curves, we determined significance levels of the corresponding linear regressions. 


\section{Results}

\section{Double simultaneous stimulation}

In a first series of experiments, the left and the right target were presented simultaneously in the double stimulation (DS) trials while the FP was varied from $-32^{\circ}$ (left) to $+32^{\circ}$ (right) of straight ahead. Figure 2 shows the frequency of selected targets (left vs. right) in the DS trials for right arm movements in animal $D$. The animal always selected the right target when the FP was straight ahead or to the left, whereas he always selected the left target when fixating at $+32^{\circ}$. Only when the FP was at $+16^{\circ}$, the monkey selected both targets equally often.

Figure 2 about here

This example demonstrates a major influence of the FP on target selection. It also reveals that simultaneous double stimulation is not an efficient way to quantify target selection preference. For instance, the animal always selected the right target for the FP at $-32^{\circ}$ and $-16^{\circ}$, even though the animal's preference to select the right target might be stronger for the FP at $-32^{\circ}$ than at $-16^{\circ}$. We therefore modified the DS task by introducing stimulus onset asynchrony (SOA) as a quantitative measure of target selection preference (see Methods).

\section{Stimulus onset asynchrony}

Figure 3A shows a series of 40 DS trials for one particular FP. Randomly interleaved single trials are not shown. Starting with simultaneous stimulus presentation on the first trial, the monkey selected the target on the right. The PEST algorithm adaptively modified the SOA, until, at about SOA $=-200 \mathrm{~ms}$ (left target first), the left and right targets were selected equally often (indicated as a horizontal line). In an off-line analysis, we fitted a logistic function to the data and defined the balanced time delay (BTD) as that SOA, for which the logistic curve crossed the 50\% line (corresponding to selecting the left and right target equally often). Figure $3 \mathrm{~B}$ shows the SOAs for the leftward (gray stars) and rightward choices (black stars) of the trials of Fig 3A. The histogram shows the selection preference as a function of SOA. Finally, the maximum-likelihood 
fitted logistic function curve is shown in black with the horizontal error bar indicating the $95 \%$ confidence interval of the BTD.

\section{Figure 3 about here}

\section{Influence of fixation position}

In each experiment, the DS task was concurrently run with FPs at $-16^{\circ}, 0^{\circ}$, and $+16^{\circ}$. Figure 4 shows a run for the saccadic response task. The three logistic functions, one for each FP, are separated along the SOA axis. The BTD for the FP straight ahead is close to zero indicating no strong preference for either target. For the FP at $-16^{\circ}$, however, the animal's preference was shifted towards the right side, as indicated by a BTD of about -200 ms (left target first). Similarly for the FP $+16^{\circ}$, the monkey's preference was shifted to the left (BTD of about $300 \mathrm{~ms}$ ). This change in preference was statistically highly significant, as indicated by the non-overlapping $95 \%$ confidence intervals for the BTDs (Fig. 4B).

\section{Figure 4 about here}

The dependence of the target selection preference on the FP was a general finding in all repetitions of the experiment and independent of the response condition (arm and eye movements). Figure 5 summarizes the result of all repetitions of each experiment obtained on six experimental days. Each histogram box (and error bar) indicates the mean (and standard deviation) of the BTD. For all response conditions, the animal had an increased preference to choose the right target when the FP was shifted to the left, and an increased preference to choose the left target when the FP was shifted to the right. To quantify this result, we predicted the BTD by a linear regression of the FP: BTD $=$ intercept + slope $* F P$. The least-square optimized coefficients are given in Table 1. For all movement conditions, in both monkeys, the resulting slope was positive and statistically highly significant $\left(\mathrm{p}<10^{-5}\right)$. For animal $G$, the slope was about $6.6 \mathrm{~ms} / \mathrm{deg}$ for the left arm, while the slope for the right arm was somewhat larger at $12.5 \mathrm{~ms} / \mathrm{deg}$. Animal $D$ had a slope for left arm movements of about $7.0 \mathrm{~ms} / \mathrm{deg}$ and $7.9 \mathrm{~ms} / \mathrm{deg}$ for the right arm. For saccadic responses, the slope was larger with about $14.4 \mathrm{~ms} / \mathrm{deg}$ for animal $G$ and 15.1 $\mathrm{ms} / \mathrm{deg}$ for animal $D$. In animal $D$ this saccadic slope was significantly larger than the left or the 
right arm responses (95\%-confidence intervals did not overlap), whereas for animal $G$, the saccadic slope was significantly larger only for the left arm (95\%-confidence intervals did overlap).

\section{Figure 5 about here}

\section{Influence of response modality}

The response modality (left arm, saccade, or right arm) had a major influence on the selection process as well. For example when monkey $G$ fixated straight-ahead (Fig. 5A), target selection preference was essentially balanced for saccadic responses (BTD $=-29 \mathrm{~ms}$ ), but was shifted to the left $(\mathrm{BTD}=113 \mathrm{~ms})$ when the animal responded with his left arm, and to the right $(\mathrm{BTD}=$ $161 \mathrm{~ms}$ ) when it responded with its right arm. In the second animal (Fig. 5B), the bias to select the target on the same side as the reaching arm was even stronger (straight-ahead FP: left arm $\mathrm{BTD}=207 \mathrm{~ms}$; right arm BTD = -207 ms). Target selection for saccadic eye movements in this animal was somewhat biased to the left (BTD: $95 \mathrm{~ms}$ ). The influence of movement modality on selection preference was present at all FPs. BTD was always larger for left arm movements than for right arm movements, and this difference was statistically significant for all FP in both animals (t-test, $\mathrm{p}<0.05$ ).

The dependence of the selection preference on the movement modality can also be seen in the linear regression model (Table 1). While the slope of the curve reflects the influence of the FP (see above), the intercept reflects the overall bias. The intercept of the linear regression for selection with left arm was significantly larger than it was for saccades (difference in intercept: $G$ $112 \mathrm{~ms}, D 82 \mathrm{~ms}$; 95\%-confidence intervals do not overlap), whereas for the right arm the intercept was significantly smaller than it was with saccades (difference in intercept: $G-92 \mathrm{~ms}, D$ $-263 \mathrm{~ms})$.

\section{Behavioral coordinate frame of target selection}

The dependence of target selection preference on the fixation position leads to the question of what underlying reference frame is being used. Target selection could take place in the coordinates of the board (board-centered), the head (head-centered), or the trunk (trunk- 
centered). In order to distinguish these different possibilities, we manipulated head position and trunk position along the horizontal axis.

\section{Variation of head position}

Figure 6 shows the results of the DS task in animal $G$ for left arm reach movements with the head in five different static horizontal head positions (HP at $-16,-8,0,+8$, and $+16^{\circ}$ on the board; Fig. 6A). Due to the constraints of the oculomotor range (horizontally $\pm 40^{\circ}$ ), the DS task in each run was limited to 3-4 FP around straight-ahead.

\section{Figure 6 about here}

Fig. 6A shows the resulting dependency of the selection preference on the FP on the board for all head positions tested on one particular day. To quantify any horizontal shift in the response curve for different head positions, we fitted a line through the data of each HP. Using the prediction of the linear fit for the HP and FP at straight-ahead as a reference line (horizontal line in Fig. 6A), we quantified for each HP the horizontal shift of the response curve by the intersection of its linear fit with this reference line (square markers). The amount of horizontal shift of the response curve with respect to head position is then given in Fig. 6B. If the response curves did not shift with HP, the graph in Fig. 6B would be flat (horizontal line), indicating a reference frame independent of HP (hence, one that was trunk- or board-centered). In contrast, if the response curve completely followed the change in HP, the graph in Fig. 6B would follow the unity line, indicating a head-centered reference frame - and this is exactly what we saw. Further, the shift coefficient $c$, which we defined as the slope of the linear regression line in Fig. 6B, indicates the relative shift of the response curve with respect to HP. In the example shown, the shift coefficient was $c=0.97$, indicating that the shift of the response curve exactly follows the change of HP.

Figure 7 summarizes the results for all movements (saccades, left and right arm movement) in both monkeys. Each condition was repeated three times and the final shift coefficient $c$ was obtained by pooling over all three repetitions. As expected, the response curves for saccadic responses shifted along with the HP ( $c=1.04$ in $G, c=1.13$ in $D$ ), indicating a head-centered reference frame. Interestingly, we also found a shift of the response curves with HP for arm 
movements. In animal $G$, the shift coefficient was 0.74 for target selection with the left arm and 0.93 for target selection with the right arm. In monkey $D$, the shift index was $c=0.79$ for right arm movements, and somewhat less, $c=0.64$, for left arm movements. In all cases, the shift coefficient was significantly larger than zero $\left(\mathrm{p}<10^{-4}\right)$.

\section{Figure 7 about here}

\section{Variation of trunk position}

In a similar fashion, we also varied the horizontal trunk position (TP), while keeping HP constant with respect to space (i.e., the board). Figure $8 \mathrm{~A}$ shows the response curves of one experimental day for left arm movements in animal $G$ (same conditions as in Fig.6) for five different static trunk positions (TP at $-16,-8,0,+8$, and $+16^{\circ}$ on the board). Because the head was always in the same position with respect to the board, each run contained the same set of FPs $\left(-16,0\right.$, and $\left.+16^{\circ}\right)$. As can be seen in Fig. 8A, we found no change in selection preference for different TPs. Just as we did with HP, we plotted the amount of horizontal shift of each response curve against TP (Fig. 8B). The flat line of this graph implies that TP does not alter target selection preference, which is also indicated by the vanishing value of the shift coefficient $(c=0.06)$.

\section{Figure 8 about here}

Figure 9 summarizes the results of the trunk variation experiments across all response conditions. As expected, target selection with saccades did not change when the trunk was rotated with the head stationary in space. The shift coefficients for the two animals (pooled across three repetitions) were $c=-0.06(G)$ and $c=-0.12(D)$. However, target selection for reaching also showed only a small change with variation in trunk position. In monkey $G$, the shift coefficient was almost negligible for left arm responses, $c=0.17$, and for right arm responses it was $c=0.03$. In monkey $D$, the shift coefficient for the trunk was somewhat larger with $c=0.29$ for the right arm and $c=0.58$ for the left arm. We therefore see a partial influence of the trunk position in animal $D$, but not in animal $G$. 


\section{Figure 9 about here}

\section{Linear model}

To further quantify the coordinate frame, we fit the linear model

$$
\mathrm{BTD}=a_{0}+a_{F P} * F P+a_{H P} * H P+a_{T P} * T P
$$

to the data of each monkey. Table 2 gives the coefficients of the linear fit in the three response conditions: saccade and left- and right-arm movements (separate regressions on the data for the head-position and the trunk-position variations gave the same results; not shown). In each fit, the constant coefficient $a_{0}$ represents the selection bias for each response modality, while $a_{F P}$ denotes the dependence on FP (as in Table 1). Further, the coefficients $a_{H P}$ and $a_{T P}$ represent the influence of the head and trunk position, respectively. In a board-centered behavioral coordinate frame, we would ideally find no influence of HP and TP, hence $a_{H P}=a_{T P}=0$ and BTD $=a_{0}+a_{F P} * F P$. In a head-centered behavioral frame of reference, the influence of the trunk would be zero, $a_{T P}=0$, and the FP would matter only with respect to the HP, hence $a_{H P}=-a_{F P}$ and BTD $=a_{0}+a_{F P} *(F P$ $-H P)$. Finally, in a trunk-centered behavioral reference frame, the influence of the head would be zero, $a_{H P}=0$, and the FP would matter only with respect to TP, leading to $a_{T P}=-a_{F P}$ and BTD $=$ $a_{0}+a_{F P} *(F P-T P)$.

To illustrate these three hypotheses, we plotted $-a_{T P} / a_{F P}$ against $-a_{H P} / a_{F P}$ in a planar graph (Fig. 10), which leads to three nearest-neighbor areas around the three ideal points (open circles) for the board-centered $(0,0)$, head-centered $(1,0)$, and trunk-centered $(0,1)$ hypothesis. For each animal, the plotted points represent the fitted coefficients of each response condition (L: left arm, R: right arm, S: saccade movement). All points remained within the nearest-neighbor region of the head-centered hypothesis, in other words, our findings can be best described in a headcentered, as opposed to a trunk-centered or board-centered behavioral reference frame. However, a partial influence of the trunk position is apparent for reach movements in animal $D$. This might indicate the existence of a combined (head-trunk) reference frame for reach target selection that differs from a purely head-centered reference frame, a result that one might expect to see for particularly large trunk excursions.

Figure 10 about here 


\section{Eye position influence}

So far, we kept the FP of the eye identical to the resting position of the arm at the beginning of the trial, and targets were presented symmetrically with respect to the eye and arm position. To investigate the role of the FP of the eye on target selection during reaching, and the potential role of the arm position on target selection with saccades, we dissociated the FP of the eye from the starting position of the arm (Fig.11). Fig. 11A shows the selection preference for animal $D$ during reaching with the left (open bars) and the right arm (filled bars). With the arm starting position constantly straight-ahead, FP of the eye was varied from a point $16^{\circ}$ above straight-ahead to points $16^{\circ}$ above the left or the right target (see icons below $\mathrm{x}$-axis). We found that target selection for reaches was biased in the direction of the FP (see Fig. 11A). For example for left arm movements, the BTD was $280 \mathrm{~ms}$ for the FP at $16^{\circ}$ to the left, $203 \mathrm{~ms}$ for the FP straightahead, and $117 \mathrm{~ms}$ for the FP at $16^{\circ}$ to the right. For the right arm, the BTD was $-84 \mathrm{~ms}$ for the $\mathrm{FP}$ at $16^{\circ}$ to the left, $-247 \mathrm{~ms}$ for FP straight-ahead, and $-240 \mathrm{~ms}$ for FP at $16^{\circ}$ to the right (linear regression slope: $-5.08 \mathrm{~ms} / \mathrm{deg}$ (left arm), $-4.89 \mathrm{~ms} / \mathrm{deg}$ (right arm); $\mathrm{p}<0.01$ ). For the second animal ( $G$, not shown), we found similar results with the same significance (linear regression slope: $7.78 \mathrm{~ms} / \mathrm{deg}$ (left arm), $-10.19 \mathrm{~ms} / \mathrm{deg}$ (right arm); $\mathrm{p}<0.01$ ).

Figure 11 about here

As a control, we also examined target selection for saccadic eye movements when the resting position of the arm was varied from $16^{\circ}$ below straight-ahead to $16^{\circ}$ below the left and right saccadic targets. The initial FP of the eye was always straight-ahead (see icons in Fig. 11B). In both animals, we did not find any significant influence of the arm resting position on the saccadic target choice ( $\mathrm{p}>0.05$ in all cases). For example in animal $D$, the BTD stayed constant at about $120 \mathrm{~ms}$ for all the different resting positions of both the left and the right arm. In animal $G$, the BTD stayed constant at about $5 \mathrm{~ms}$ (data not shown). Taken together, these findings show that target selection for an arm movement is influenced by the FP of the eye, whereas target selection for a saccadic eye movement is not influenced by the resting position of the arm. 


\section{Response times}

We also examined the response times (RT) and movement times (MT) for the reach and saccade response tasks in both animals. Figure 12 shows a summary of the RTs (open bars) and MTs (filled bars) for the four trial conditions (L: single target to the left, R: single target to the right, CL double stimulation with choice of the left target, CR: double stimulation with choice of the right target) for the three different FPs that we tested. For reach movements, the RT was $585 \pm 33 \mathrm{~ms}$ (mean $\pm \mathrm{SD}$ ) for animal $D$ and $551 \pm 33 \mathrm{~ms}$ for animal $G$ across all conditions. MT was also fairly constant with a mean of $257 \pm 23 \mathrm{~ms}$ in animal $D$ and $232 \pm 25 \mathrm{~ms}$ in animal $G$.

For saccades, the MT was constant with a mean of $73 \pm 5 \mathrm{~ms}($ animal $D$ ) and $82 \pm 8 \mathrm{~ms}$ (animal $G)$ across all conditions. Saccadic RT, however, did vary across different FP and trial conditions. For saccades with the FP straight-ahead and for saccades made towards the center, the RT was short ( $D: 244 \pm 34 \mathrm{~ms}, G 213 \pm 27 \mathrm{~ms}$ ). However, the RT was substantially longer for the DS trials and single trials toward the periphery in the off-center FPs ( $D: 396 \pm 63 \mathrm{~ms}, G: 362 \pm 33 \mathrm{~ms}$ ). This difference can be explained by the larger BTD in the off-center DS trials (in the order of 200ms, see Fig. 5) as opposed to the lower BTD $(<100 \mathrm{~ms})$ for the DS trials in the straight-ahead FP. In the reach responses, we do not see this effect despite similar differences in BTD across different FPs (Fig. 5). This might be due to the fact that the RT for saccades is much faster (in the order of $200-400 \mathrm{~ms}$ ) than for reach movements (in the order of $600 \mathrm{~ms}$ ).

Figure 12 about here 


\section{Discussion}

\section{Summary of results}

In this study, we examined how the selection of targets for saccade and reach movements is influenced by the location of the target and what coordinate frame underlies the target selection process. We trained monkeys in a paradigm in which targets were presented on opposite sides of a FP (Fig. 1). Simultaneous presentation of two targets on probe trials revealed that for many target locations the animal strongly preferred one target over the other (Fig. 2). To quantify this preference, we presented the non-preferred target first at a variable SOA until the left and right targets were selected equally often (Fig. 3A). The balanced time delay then provides a reliable measure of the degree of preference for one target over the other (Fig. 3B).

We found that the target selected for movements of the eye and arm both depend on fixation position. For all movements, the preference for the left target increased when both targets were presented on the right and decreased when both targets were presented on the left (Fig. 4, 5 and Table 1). Further, we observed a bias for the left targets when the left arm was used and a bias for right targets when the right arm was used (Fig. 5 and Tab. 1). To determine whether targets were being selected in a head-centered or a trunk-centered reference frame, we systematically varied head and trunk position (Fig. 6-8). We found a large change in target preference when the head was rotated (Fig. 6,7), but only a small change when the trunk was rotated (Fig. 8, 9). Using a linear model, we found that a head-centered reference frame captured our findings much better than a trunk-centered or a board-centered representation (Fig. 10 and Tab. 2). While this finding was expected for saccades, the strong dependence of target selection for reach movements on head position, and the relative small dependence of the trunk position, in this task was somewhat counterintuitive. Using a linear model, we found that a head-centered reference frame captured our findings better than a trunk-centered or a board-centered representation (Fig. 10 and Tab. 2). This was true even for animal $D$, where a partial influence of the trunk position on reach target selection was observed. Finally, we found that the selection of reach targets was strongly 
dependent on fixation position, whereas the selection of saccade targets did not depend on the position of the arm during the task (Fig. 11).

\section{Comparison of results with psychophysical and clinical evidence}

When a reach movement is planned to a visual stimulus, the target direction has ultimately to be transformed from a visual reference frame of the retinal image to a trunk-centered representation that guides the execution of the arm movement (Soechting and Flanders, 1991; Desmurget et al., 1998). The coordinate frame, in which the reach target is selected, could be eye, head-, or body-centered, or even in some combination of these frames (Flanders and Soechting, 1995; Soechting and Flanders, 1995). In everyday life, when we reach out to pick up an object we often look at the intended target. Even when we do not, we shift our attention to the target in order to generate a reach towards it (Deubel et al., 1997; Deubel et al., 1998). As we mentioned in the Introduction, there is evidence that attention and eye movement control share much of the same neural circuitry (Rizzolatti et al., 1987; Deubel and Schneider, 1996; Corbetta et al., 1998). We might expect therefore that the selection of a target for a reaching movement would share the same underlying frame of reference employed by the saccadic system. Selecting the target in this saccadic frame of reference provides a useful prelude to converting this representation of the target into the required coordinates for the arm movement. Our evidence supports this conjecture: target selection for reaching, like target selection for saccades, is modulated by eye position, and not mainly by body position, as one might have thought. In other words, in this task, target selection for both eye and limb movements occur in a similar reference frame (Gielen et al., 1984; Frens and Erkelens, 1991; McIntyre et al., 1997; Soechting et al., 2001).

Our findings are consistent with previous results: Neggers and Bekkering (2000, 2001), who examined the coordination of eye and arm movements in a sequential reaching task, showed that ocular gaze is anchored to the target of an ongoing pointing movement until the movement is finished, even when the moving arm is not visible. This "yoking" of eye movements to the slower movements of the arm seems to imply, quite in agreement with our findings, that a common control mechanism links the eye and arm effector systems and that the planning and execution of reaching movements depends on the reference frame of the visual system (see also McIntyre et 
al., 1997; Soechting et al., 2001). Such a system could be particularly useful for the manipulation of objects (Johansson et al., 2001). A similar conclusion was attained in a study where subjects quickly fixated and pointed at unexpectedly presented eccentric targets (Frens and Erkelens, 1991). When a gap was introduced between extinction of a fixation point and target presentation, subjects were forced to guess and the error rate in the initial movement direction of saccade and hand movements increased to about 50\%. Nevertheless, saccade and hand movements were always made in the same direction, which suggests that target selection for eye and hand movements made on the basis of cognitive information share a common mechanism. Similar conclusions were also drawn from a single- and double-step tracking task (Gielen et al., 1984). Finally, Fisk and Goodale (1985) studied the latency and kinematics of eye and arm movements in an unrestricted looking and pointing task, where they found that the saccade latencies during looking and pointing to a particular target were influenced by which arm was used. It was suggested that reaching toward a target under visual control involves a common integration of both eye and arm movements.

To interpret our finding of a head-centered behavioral reference frame, one has to bear in mind that saccade and reach targets were always presented at the same distance to the initial eye and arm position in our task. This controls for any eye-centered position effects on saccade target selection and for any hand-centered position effects on reach target selection (Tipper et al., 1992; Tipper et al., 1998). All stimulus conditions being equal (targets at equal distance to the FP, equal stimulus intensity, and equal amounts of reward associated with each target), the finding of a head-centered reference frame for saccade target selection was not unexpected. Saccade targets are preferred that bring the eye back to the head-centered midline, or in other words, eye position introduces a bias for the selection of saccade targets. For reach movements, however, the strong dependence of target selection on head position was surprising. Reach movements essentially fall into the same eye-position bias for target selection as saccades. Furthermore, when we dissociated the initial eye and arm position in our experiment, an eye-position effect for reach target selection was observed that favored targets that were closer to the FP. In contrast, arm position had no effect on saccade target selection. This result reveals an influence of the eyes on the planning of reach movements. 
Target selection during visual double stimulation is compromised after parietal and frontal brain lesions. In animal studies, temporary inactivation of frontal and parietal areas can produce a condition called visual extinction, in which subjects are unable to perceive the contra-lesional of two simultaneously presented visual stimuli, whereas each stimulus can readily be detected if presented singly (Li and Andersen, 1997; Schiller and Chou, 1998). Patients suffering from parietal or frontal lesions often also show this effect (for a review see Heilman et al., 1993). The severity of extinction strongly depends on the spatial stimulus location with much stronger effects when both stimuli are presented in the contralesional hemifield, while extinction is much weaker or absent when both stimuli are presented in the ipsilesional hemifield (Di Pellegrino and De Renzi, 1995; Smania et al., 1996), The fact that target selection in the intact primate and visual extinction are both influenced by the spatial location of the stimuli indicates that a common neural network might be involved in both effects. Furthermore, studies aiming to determine the underlying reference frame of extinction found, in correspondence with our results, that both the retinotopic and the hemispatial position of the extinguished stimulus determined the severity of visual extinction (Rapcsak et al., 1987; Kooistra and Heilman, 1989; Smania et al., 1996).

\section{Neuronal correlates for target selection}

The parietal lobe plays an important role for the coordination of eye and hand movements, which is evident from experimental and clinical lesions. In an experiment where posterior parietal areas were temporarily inactivated by cooling, the coordination of reach and eye movements were disrupted (Stein, 1978). In humans, a striking case report of disrupted eye-hand coordination was made about a patient with bilateral parietal atrophy, who was unable to reach to targets to which she was not allowed to look and consistently mis-reached to the location of where her eyes were fixating (Carey et al., 1997). These and other studies are consistent with the central roles of the parietal cortex in space representation for visuo-motor actions, such as reaching, pointing, grasping, and looking (Goodale and Milner, 1992; Goodale and Haffenden, 1998).

Electrophysiological studies in the monkey have identified distinguishable subregions in the PPC for the high-level, or cognitive, planning of saccades, hand reaching, and grasping 
(Mountcastle et al., 1975; Gnadt and Andersen, 1988; Sakata et al., 1995; Snyder et al., 1997). Neurons in the PPC encode the target location of upcoming movements in an eye-centered coordinate frame for both the planning of saccades and for arm reach movements (Snyder et al., 1997; Batista et al., 1999; Buneo et al., 2002). This might provide a particularly simple way to facilitate the coordination of eye and hand movements (Scherberger and Andersen, in press). The activity of these neurons, though eye-centered, is modulated by the eye and arm position in space (Andersen et al., 1985; Andersen et al., 1990; Buneo et al., 2002). These eye- or arm-position gain fields implement a common and distributed representation of space that allows to read out target coordinates in multiple coordinate frames, e.g., head-centered, at subsequent processing stages (Zipser and Andersen, 1988; Andersen et al., 1997; Xing and Andersen, 2000). Our finding of a head-centered behavioral reference frame for target selection for arm and eye movements is therefore consistent with current concepts of space representation in the PPC.

Electrophysiological studies aiming to understand how the brain arrives at decisions suggest that decision-making is a distributed process that is reflected in the neuronal activity of many brain areas including the frontal cortex, the intraparietal area (LIP), and the superior colliculus, (Shadlen and Newsome, 1996; Platt and Glimcher, 1998; Horwitz and Newsome, 1999; Kim and Shadlen, 1999; Coe et al., 2002; for a review see: Schall, 2001). Preliminary electrophysiological recordings during target selection for reach movements in the parietal reach region (PRR) showed a correlation between the firing rate of individual cells and the reach choice (Scherberger and Andersen, 2001), which confirms that the PPC is participating in the decision process for reach target selection. It remains to be seen, however, to what extent the activity in LIP and PRR dissociates for saccade and reach decisions. We are currently examining whether LIP and PRR arrive at decisions for saccades and reaches in an independent fashion, whether LIP plays a more executive role in decision making for PRR and reaches, or whether frontal lobe structures exert control over both these parietal areas. 


\section{Acknowledgement}

We would like to thank Larry Snyder for support on the recording software, Betty Gillikin and

Kelsie Weaver for animal care, Cierina Marks for administrative help, and Viktor Shcherbatyuk for technical support. This work was supported by the Christopher Reeve Paralysis Foundation, a Caltech Gosney Fellowship, and the National Eye Institute. 


\section{References}

Andersen RA, Essick GK, Siegel RM (1985) Encoding of spatial location by posterior parietal neurons. Science 230:456-458.

Andersen RA, Snyder LH, Bradley DC, Xing J (1997) Multimodal representation of space in the posterior parietal cortex and its use in planning movements. Annu Rev Neurosci 20:303330.

Andersen RA, Bracewell RM, Barash S, Gnadt JW, Fogassi L (1990) Eye Position Effects On Visual, Memory, and Saccade-Related Activity in Areas Lip and 7a of Macaque. Journal of Neuroscience 10:1176-1196.

Ballard DH, Hayhoe MM, Pelz JB (1995) Memory representations in natural tasks. J Cognit Neurosci 7:66-80.

Ballard DH, Hayhoe MM, Li F, Whitehead SD (1992) Hand-eye coordination during sequential tasks. Philos Trans R Soc Lond B Biol Sci 337:331-338; discussion 338-339.

Batista AP, Buneo CA, Snyder LH, Andersen RA (1999) Reach plans in eye-centered coordinates. Science 285:257-260.

Buneo CA, Jarvis MR, Batista AP, Andersen RA (2002) Direct visuomotor transformations for reaching. Nature 416:632-636.

Carey DP, Coleman RJ, Della Sala S (1997) Magnetic misreaching. Cortex 33:639-652.

Carpenter RH (1988) Movement of the eyes (2nd ed). London: Pion Limited.

Coe B, Tomihara K, Matsuzawa M, Hikosaka O (2002) Visual and anticipatory bias in three cortical eye fields of the monkey during an adaptive decision-making task. J Neurosci 22:5081-5090.

Corbetta M, Akbudak E, Conturo TE, Snyder AZ, Ollinger JM, Drury HA, Linenweber MR, Petersen SE, Raichle ME, Van Essen DC, Shulman GL (1998) A common network of functional areas for attention and eye movements. Neuron 21:761-773.

Desmurget M, Pelisson D, Rossetti Y, Prablanc C (1998) From eye to hand: planning goaldirected movements. Neurosci Biobehav Rev 22:761-788. 
Deubel H, Schneider WX (1996) Saccade target selection and object recognition: evidence for a common attentional mechanism. Vision Res 36:1827-1837.

Deubel H, Shimojo S, Paprotta I (1997) Line motion illusion and selection-for-action: Further evidence for the coupling of visual attention and the preparation of goal-directed movements. Investigative Ophthalmology and Visual Science 38:5381-5381.

Deubel H, Schneider WX, Paprotta I (1998) Selective dorsal and ventral processing: evidence for a common attentional mechanism in reaching and perception. Visual Cognition 5:81-107.

Di Pellegrino G, De Renzi E (1995) An experimental investigation on the nature of extinction. Neuropsychologia 33:153-170.

Fisk JD, Goodale MA (1985) The organization of eye and limb movements during unrestricted reaching to targets in contralateral and ipsilateral visual space. Exp Brain Res 60:159-178.

Flanders M, Soechting JF (1995) Frames of Reference For Hand Orientation. Journal of Cognitive Neuroscience 7:182-195.

Frens MA, Erkelens CJ (1991) Coordination of hand movements and saccades: evidence for a common and a separate pathway. Exp Brain Res 85:682-690.

Gescheider GA (1997) Psychophysics: The Fundamentals (third edition). Mahwah, NJ: Lawrence Erlbaum Associates.

Gielen CC, Heuvel PJM, van Gisbergen JA (1984) Coordination of fast eye and arm movements in a tracking task. Exp Brain Res 56:154-161.

Gnadt JW, Andersen RA (1988) Memory related motor planning activity in posterior parietal cortex of macaque. Exp Brain Res 70:216-220.

Goodale MA, Milner AD (1992) Separate visual pathways for perception and action. Trends Neurosci 15:20-25.

Goodale MA, Haffenden A (1998) Frames of reference for perception and action in the human visual system. Neurosci Biobehav Rev 22:161-172.

Heilman KM, Watson RT, Valenstein E (1993) Neglect and related disorders. In: Cinical Neuropsychology (third edition) (Heilman KM, Valenstein E, eds), pp 279-336. New York, Oxford: Oxford University Press. 
Horwitz GD, Newsome WT (1999) Separate Signals for Target Selection and Movement Specification in the Superior Colliculus. Science 284:1158-1161.

Johansson RS, Westling G, Backstrom A, Flanagan JR (2001) Eye-hand coordination in object manipulation. J Neurosci 21:6917-6932.

Judge SJ, Richmond BJ, Chu FC (1980) Implantation of magnetic search coils for measurement of eye position: an improved method. Vision Res 20:535-538.

Kim JN, Shadlen MN (1999) Neural correlates of a decision in the dorsolateral prefrontal cortex of the macaque. Nat Neurosci 2:176-185.

Kooistra CA, Heilman KM (1989) Hemispatial visual inattention masquerading as hemianopia. Neurology 39:1125-1127.

Land M, Mennie N, Rusted J (1999) The roles of vision and eye movements in the control of activities of daily living. Perception 28:1311-1328.

Li CS, Andersen RA (1997) Lesion of a macaque parietal area produces visual extinction in an egocentric framework. Invest Ophthalmol Vis Sci 38, suppl.:655.

Macmillan NA, Creelman CD (1991) Detection theory: a user's guide. Cambridge: Cambridge University Press.

McIntyre J, Stratta F, Lacquaniti F (1997) Viewer-centered frame of reference for pointing to memorized targets in three-dimensional space. J Neurophysiol 78:1601-1618.

Mountcastle VB, Lynch JC, Georgopoulos A, Sakata H, Acuna C (1975) Posterior parietal association cortex of the monkey: command functions for operations within extrapersonal space. J Neurophysiol 38:871-908.

Neggers SF, Bekkering H (2000) Ocular gaze is anchored to the target of an ongoing pointing movement. J Neurophysiol 83:639-651.

Neggers SF, Bekkering H (2001) Gaze anchoring to a pointing target is present during the entire pointing movement and is driven by a non-visual signal. J Neurophysiol 86:961-970.

Platt ML, Glimcher PW (1997) Responses of intraparietal neurons to saccadic targets and visual distractors. Journal of Neurophysiology 78:1574-1589.

Platt ML, Glimcher PW (1998) Response fields of intraparietal neurons quantified with multiple saccadic targets. Experimental Brain Research 121:65-75. 
Rapcsak SZ, Watson RT, Heilman KM (1987) Hemispace-visual field interactions in visual extinction. Journal Of Neurology, Neurosurgery And Psychiatry 50:1117-1124.

Rizzolatti G, Riggio L, Dascola I, Umilta C (1987) Reorienting attention across the horizontal and vertical meridians: evidence in favor of a premotor theory of attention. Neuropsychologia 25:31-40.

Robinson DA (1975) A quantitative analysis of extraoclular mscle cooperation and squint. Investigative Ophthalmology 14:801-825.

Ruete CGT (1855) Lehrbuch der Ophthalmologie fuer Aertze und Studirende. (2 vols.). Braunschweig: Vieweg.

Sakata H, Taira M, Murata A, Mine S (1995) Neural mechanisms of visual guidance of hand action in the parietal cortex of the monkey. Cereb Cortex 5:429-438.

Schall JD (2001) Neural basis of deciding, choosing and acting. Nat Rev Neurosci 2:33-42.

Scherberger H, Andersen RA (2001) Neural activity in the posterior parietal cortex during decision processes for generating visually-guided eye and arm movements in the monkey. SocNeurosciAbst 27: 237.8.

Scherberger H, Andersen RA (in press) Senosorimotor transformations. In: The Visual Neurosciences (Chalupa LM, Werner JS, eds). Cambridge, MA: MIT Press.

Scherberger H, Goodale MA, Andersen RA (1999) Reaching and saccadic target selection both follow a head- rather than trunk-centered reference frame during visual double simultaneous stimulation in the monkey. SocNeurosciAbst 25: 873.9.

Schiller PH, Chou IH (1998) The effects of frontal eye field and dorsomedial frontal cortex lesions on visually guided eye movements. Nature Neuroscience 1:248-253.

Shadlen MN, Newsome WT (1996) Motion perception: seeing and deciding. Proc Natl Acad Sci U S A 93:628-633.

Smania N, Martini MC, Prior M, Marzi CA (1996) Input and response determinants of visual extinction: A case study. Cortex 32:567-591.

Snyder LH, Batista AP, Andersen RA (1997) Coding of intention in the posterior parietal cortex. Nature 386:167-170. 
Soechting JF, Flanders M (1991) Arm movements in three-dimensional space: computation, theory, and observation. Exerc Sport Sci Rev 19:389-418.

Soechting JF, Flanders M (1992) Moving in three-dimensional space: frames of reference, vectors, and coordinate systems. Annu Rev Neurosci 15:167-191.

Soechting JF, Flanders M (1995) Psychophysical approaches to motor control. Curr Opin Neurobiol 5:742-748.

Soechting JF, Engel KC, Flanders M (2001) The Duncker illusion and eye-hand coordination. J Neurophysiol 85:843-854.

Stein J (1978) Effects of parietal lobe cooling on manipulative behaviour in the conscious monkey. In: Active touch (Gordon G, ed), pp 79-90. New York: Pergamon.

Taylor MM, Creelman CD (1967) PEST: Efficient estimates on probability functions. Journal of the Acoustical Society of America 41:782-787.

Tipper SP, Lortie C, Baylis GC (1992) Selective reaching: evidence for action-centered attention. J Exp Psychol Hum Percept Perform 18:891-905.

Tipper SP, Howard LA, Houghton G (1998) Action-based mechanisms of attention. Philos Trans R Soc Lond B Biol Sci 353:1385-1393.

Treutwein B (1995) Adaptive Psychophysical Procedures. Vision Research 35:2503-2522.

Weymouth FW (1958) Visual sensory units and the minimal angle of resolution. Am J Ophthalmol 46:102-113.

Xing J, Andersen RA (2000) Models of the posterior parietal cortex which perform multimodal integration and represent space in several coordinate frames. J Cogn Neurosci 12:601614.

Yarbus A (1967) Eye movements and vision [translated from Russian by Haigh B]. New York: Plenum Press.

Zipser D, Andersen RA (1988) A back-propagation programmed network that simulates response properties of a subset of posterior parietal neurons. Nature 331:679-684. 


\section{Figure captions}

\section{Figure 1}

Experimental setup and paradigm. A: Animal seated in front of a reach board with buttons spaced $16^{\circ}$, each button containing a red and a green LED light. Animal is fixation and touching a fixation point (FP), then one or two targets (T) appear on either side. B: Location of the FP (bold square) and targets (arrow) can vary from $32^{\circ}$ to the left (I) to $32^{\circ}$ to the right (V). C: ReachParadigm. First, a fixation point (FP) appears, which has to be fixated and touched. Then either one or two targets are illuminated in green at $16^{\circ}$ on either side of the FP. Percentages indicate the distribution of concurrently running trials. In saccade trials (not shown), targets are illuminated in red.

\section{Figure 2}

Selection preference for double simultaneous stimulation. Frequency of left (open bars) and right target selection (filled bars) of right arm reaches for different horizontal fixation positions.

\section{Figure 3}

Target selection with stimulus onset asynchrony (SOA). A: Left (gray squares) and right target selections (black squares) in a block of 40 trials (abscissa). Ordinate: SOA (left target onset right target onset, positive values indicate that the right target appears first). SOA is adapted using the adaptive procedure PEST (see Methods) and converges toward the balanced time delay (BTD), for which left and right targets are selected equally often (horizontal line). B: Logistic function fit. *: SOA of data from A for leftward (bottom margin) and rightward choices (top margin). Bars: histogram of selection preference. Sigmoid curve: Maximum-likelihood estimate of the logistic distribution (see Methods). Vertical line: balanced time delay (BTD), defined as the SOA for which the logistic distribution reaches half maximum (50\% rightward choices). 


\section{Figure 4}

Target selection preference for different fixation positions. A: Logistic function fits for target selections with the fixation point (FP) at straight-ahead and at $16^{\circ}$ to the left and the right. *: SOA of data points for leftward (bottom margin) and rightward choices (top margin). Logistic functions (sigmoid curves) and BTD (vertical lines) fitted separately for each FP. B: Target selection preference (in terms of BTD) for different FP (error bars: 95\% confidence interval).

\section{Figure 5}

Summary: Target selection preference. Results of animal $G$ (panel A) and animal $D$ (panel B) for left arm responses (open bars), saccades (light bars) and right arm responses (filled bars). Fixation position was varied from straight ahead to $16^{\circ}$ to the left and the right (abscissa). Target selection preference is expressed as BTD (see Fig. 4). Error bars: 95\% confidence interval.

\section{Figure 6}

Variation of head position. Left arm movements in animal $G$. A: Monotonic change of selection preference (BTD) with fixation position shown for the head at straight-ahead (solid line) and at $8^{\circ}$ (dotted lines) and $16^{\circ}$ (dashed lines) to either side while the trunk was kept stationary in space. Horizontal line: Reference line for the BTD with the head and FP straight-ahead. Squares: intersection of response curves with reference line. B: Shift of response curves (squares in A) plotted against the change of head position (HP). Data points scatter around the unity line, indicating a response shift that matches the change of HP. c: shift coefficient, defined as the regression slope of the response shift and the change of HP. Dotted lines: 95\% confidence interval of the regression slope.

\section{Figure 7}

Summary: variation of head position. Response shifts vs. head position for saccade, left arm and right arm responses for each animal $(G, D)$. Each panel plots the response shift (see Fig. 6) vs. the change of head position (HP). Shift coefficients (c) close to 1 indicate that the response curves shift along with the head. This was true for saccades, but also for left and right arm movements. 


\section{Figure 8}

Variation of trunk position. Left arm movements in animal G. A: Monotonic change of selection preference (BTD) with fixation position shown for the trunk at straight-ahead (solid line) and at $8^{\circ}$ (dotted lines) and $16^{\circ}$ (dashed lines) to either side while the head was kept stationary in space. Horizontal line: Reference line for the BTD with the trunk and FP straight-ahead. Squares: intersection of response curves with reference line. B: Shift of response curves (squares in A) plotted against the change of trunk position (TP). Data points scatter around the constant-zero line, indicating that the response curves in A do not change with trunk position TP. c: shift coefficient, defined as the regression slope of the response shift and the change of TP. Dotted lines: 95\% confidence interval of the regression slope.

\section{Figure 9}

Summary: variation of trunk position. Response shifts vs. trunk position for saccade, left arm and right arm responses for each animal $(G, D)$. Each panel plots the response shift (see Fig. 7) vs. the change of trunk position (TP). Shift coefficients (c) close to 0 indicate that the response curves do not shift along with the trunk. This was true for saccades, but also for most left and right arm movements, however, note that animal $D$ showed a significant trunk movement dependency, in particular when responding with its left arm.

\section{Figure 10}

Classification of coordinate frames on the basis of the coefficients of the linear model: $B T D=a_{0}+a_{F P} * F P+a_{H P} * H P+a_{T P} * T P$. Abscissa: coefficient ratio $-a_{H P} / a_{F P}$, ordinate: coefficient ratio $-a_{T P} / a_{F P}$. Lines indicate nearest neighbor regions around the points $(0,0),(1,0)$, and $(0,1)$ that indicate a board-centered, head-centered, and trunk-centered reference frame, respectively (see Result section). Markers indicate the fitted response coefficients for left arm $(\mathrm{L})$, right arm (R), and saccade responses (S) for animal $G$ and $D$. All response conditions fall into the head-centered region. 


\section{Figure 11}

Eye position influences on reach responses. A: Selection preferences for reach responses starting at straight-ahead, but with the eye either fixating above straight-ahead (middle bars), above the left target (left bars), or above the right target (right bars; see icons at the bottom). Selection preferences for left arm (open bars) and right arm responses (filled bars) are both modulated and shifted toward the resting position of the eye. B: Control experiment examining the selection preferences of saccades from straight-ahead with the left arm (open bars) or right arm (filled bars) resting beneath straight-ahead (middle bars), the left target (left bars), or the right target (right bars; see icons at the bottom). Target selection for saccades is not influenced by the position of the resting arm.

\section{Figure 12}

Reaction times and movement times. Reaction times (light bars) and movement times (dark bars) for single trials to the left $(\mathrm{L})$ and right $(\mathrm{R})$ and for choice trials to the left $(\mathrm{CL})$ and right $(\mathrm{CR})$ for the fixation position at straight ahead and at $16^{\circ}$ to the left and right. Different panels show different response modalities (left arm, right arm, saccades) for the two animals $(G, D)$. 


\section{Table captions}

\section{Table 1}

Title: Linear regression of selection preference (BTD) on fixation position (FP): BTD = intercept + slope * FP

Explanatory footnote: Values are least-square fitted coefficients with $95 \%$ confidence interval in parenthesis. Coefficients are given separately for both animals $(G, D)$ and for left arm, saccade, and right arm movements. Linear regression model: BTD $=$ intercept + slope $*$ FP with coefficients intercept (in $m s$ ) and slope (in $m s / d e g$ ). $\mathrm{r}^{2}$ : coefficient of determination.

\section{Table 2}

Title: Linear regression of selection preference (BTD) on fixation position (FP), and its dependence on head and trunk position: $\mathrm{BTD}=\mathrm{a}_{0}+\mathrm{a}_{\mathrm{FP}} * \mathrm{FP}+\mathrm{a}_{\mathrm{HP}} * \mathrm{HP}+\mathrm{a}_{\mathrm{TP}} * \mathrm{TP}$

Explanatory footnote: Values are least-square fitted coefficients, given separately for both animals $(G, D)$ and for left arm, saccade, and right arm movements. Linear regression model: $\mathrm{BTD}=\mathrm{a}_{0}+\mathrm{a}_{\mathrm{FP}} * \mathrm{FP}+\mathrm{a}_{\mathrm{HP}} * \mathrm{HP}+\mathrm{a}_{\mathrm{TP}} * \mathrm{TP}$ with coefficients $\mathrm{a}_{0}$ indicating a constant bias, $\mathrm{a}_{\mathrm{FP}}$ indicating the influence of the fixation position (FP), and $\mathrm{a}_{\mathrm{HP}}$ and $\mathrm{a}_{\mathrm{TP}}$ indicating the influence of the head (HP) and trunk position (TP). Units: $\mathrm{a}_{0}$ in $m s ; \mathrm{a}_{\mathrm{FP}}, \mathrm{a}_{\mathrm{HP}}$, and $\mathrm{a}_{\mathrm{TP}}$ in $m s / d e g . \mathrm{r}^{2}$ : coefficient of determination. *, values significantly different from zero $(\mathrm{p}<0.05)$. 
JN-00883-2002.R1, page 33

\section{Tables}

\section{Table 1}

\begin{tabular}{llllll}
\multicolumn{5}{c}{ intercept } & $\mathrm{r}^{2}$ \\
$G$ & & & & \\
Left arm & 113 & $(99,126)$ & 6.59 & $(5.56,7.63)$ & 0.84 \\
Saccades & 1 & $(-19,21)$ & 14.37 & $(12.86,15.88)$ & 0.92 \\
Right arm & -91 & $(-116,-66)$ & 12.51 & $(10.59,14.43)$ & 0.85 \\
$D$ & & & & & \\
Left arm & 183 & $(166,200)$ & 6.98 & $(5.67,8.29)$ & 0.79 \\
Saccades & 101 & $(83,118)$ & 15.05 & $(13.71,16.39)$ & 0.94 \\
Right arm & -162 & $(-185,-138)$ & 7.86 & $(6.06,9.66)$ & 0.72
\end{tabular}


JN-00883-2002.R1, page 34

\section{Table 2}

$\begin{array}{lllll} & \mathrm{a}_{0} & \mathrm{a}_{\mathrm{FP}} & \mathrm{a}_{\mathrm{HP}} & \mathrm{a}_{\mathrm{TP}} \\ G & & & & \\ \text { Left arm reach } & 112.93^{*} & 6.04^{*} & -4.72^{*} & -0.91 \\ \text { Saccade } & 7.83 & 15.23^{*} & -16.17^{*} & 1.10 \\ \text { Right arm reach } & -73.50^{*} & 11.9^{*} & -11.44^{*} & -0.27 \\ D & & & & \\ \text { Left arm reach } & 158.21^{*} & 6.99^{*} & -4.53^{*} & -4.11^{*} \\ \text { Saccade } & 96.22^{*} & 14.86^{*} & -16.39^{*} & 1.86^{*} \\ \text { Right arm reach } & -157.91^{*} & 7.61^{*} & -6.07^{*} & -2.63^{*}\end{array}$


A

B

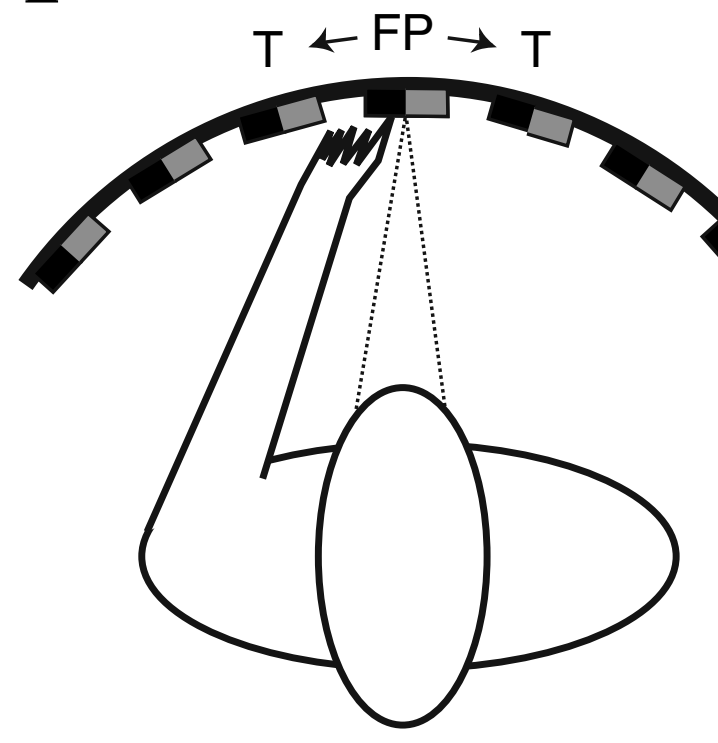

$\mathrm{T} \leftarrow \mathrm{FP} \rightarrow \mathrm{T}$

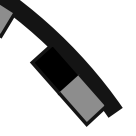

Targets: $\quad-48-32-160163248^{\circ}$

$1 \quad \square+\square \rightarrow \square \quad \square \quad \square \quad-32^{\circ}$

II $\square \square \leftarrow \square \rightarrow \square \quad \square \quad \square \quad-16^{\circ}$

III $\square \square \square \leftarrow \square \rightarrow \square \quad \square \quad \square \quad 0^{\circ}$

IV $\square \square \square \square \leftarrow \square \rightarrow \square \square \square$

V $\square \square \square \square \square<\square \rightarrow \square \quad 32^{\circ}$
C

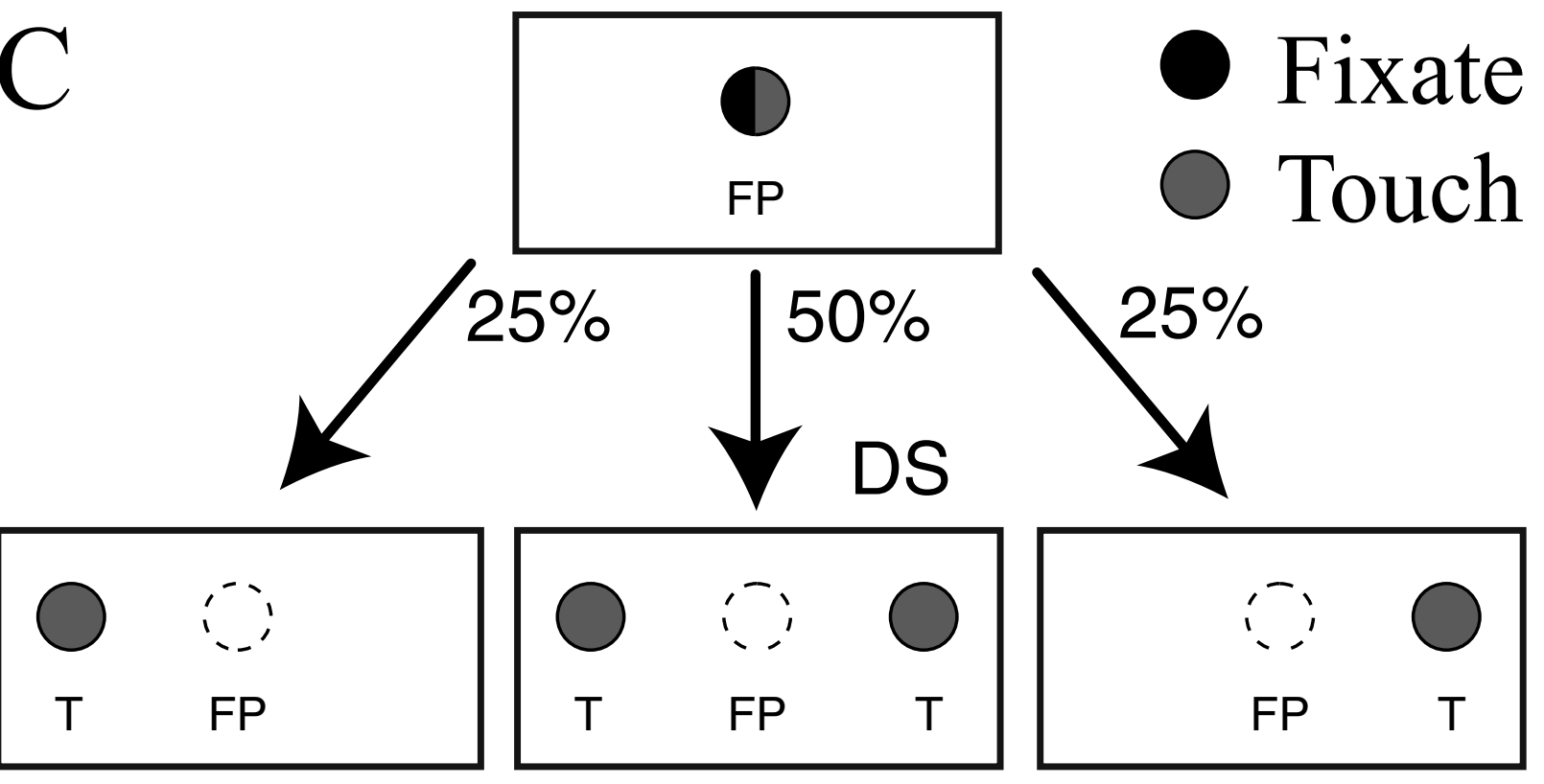




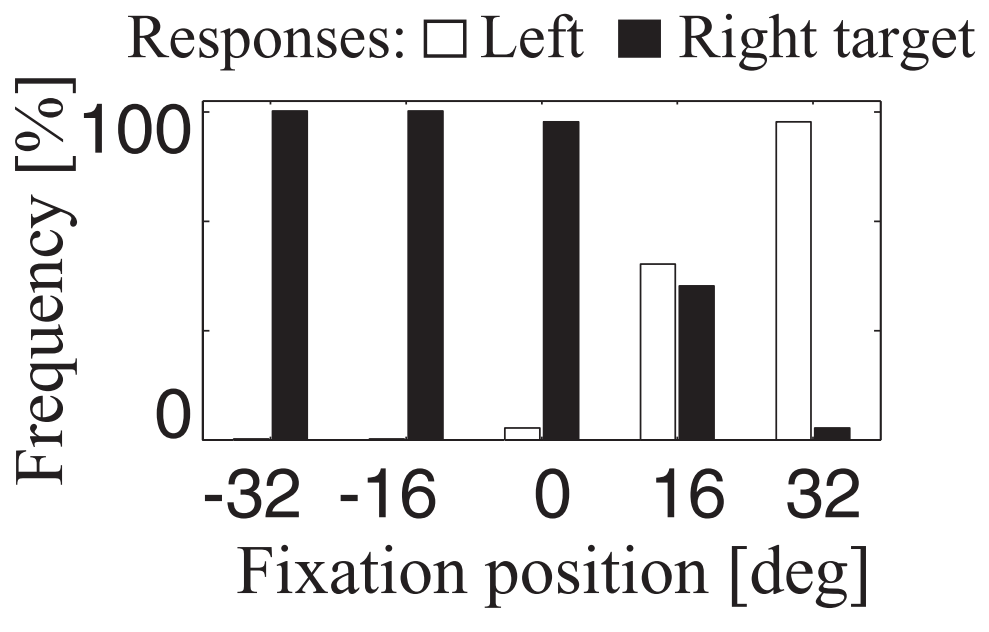

Scherberger et al, Figure 2 


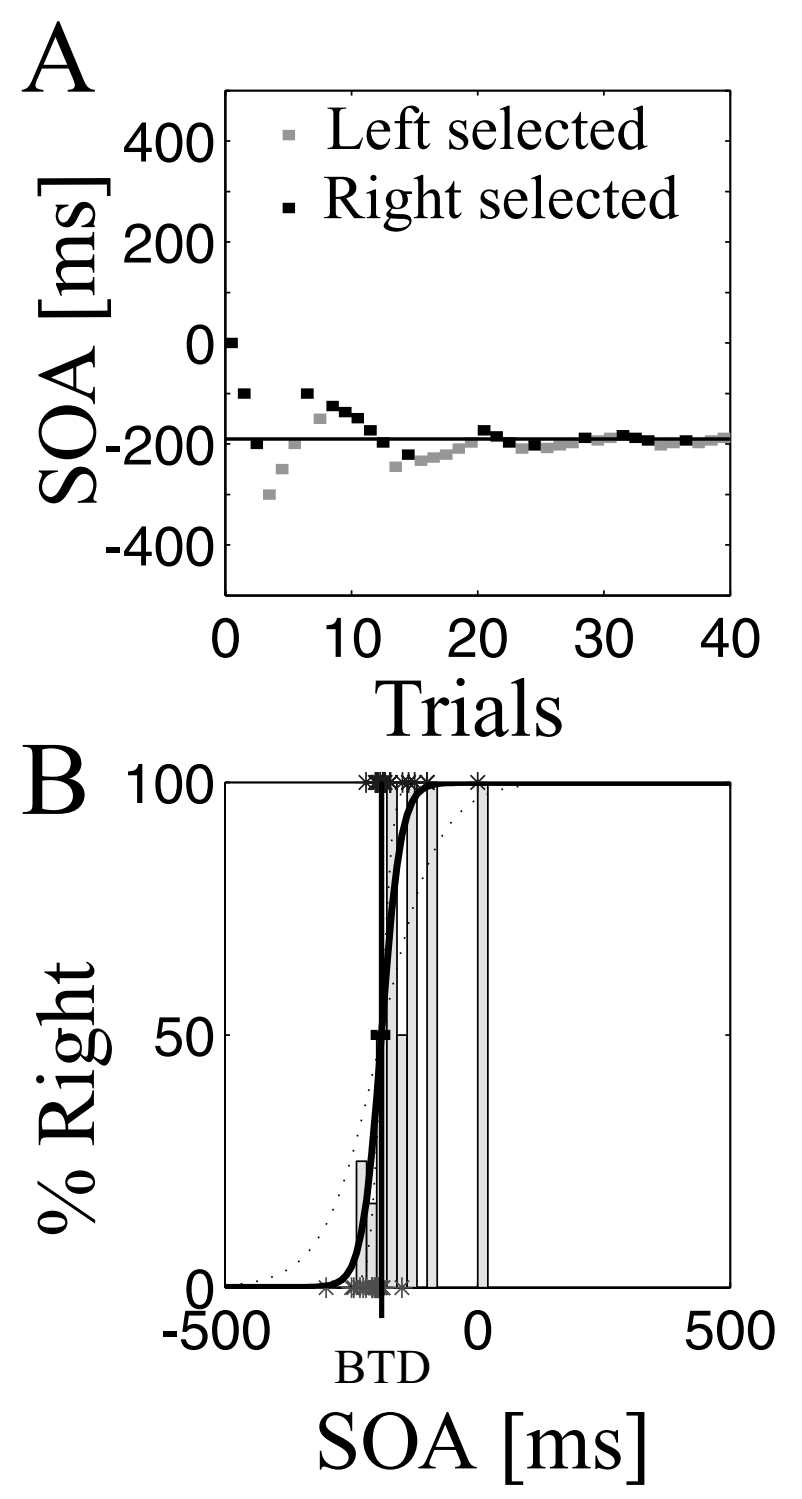

Scherberger et al, Figure 3 


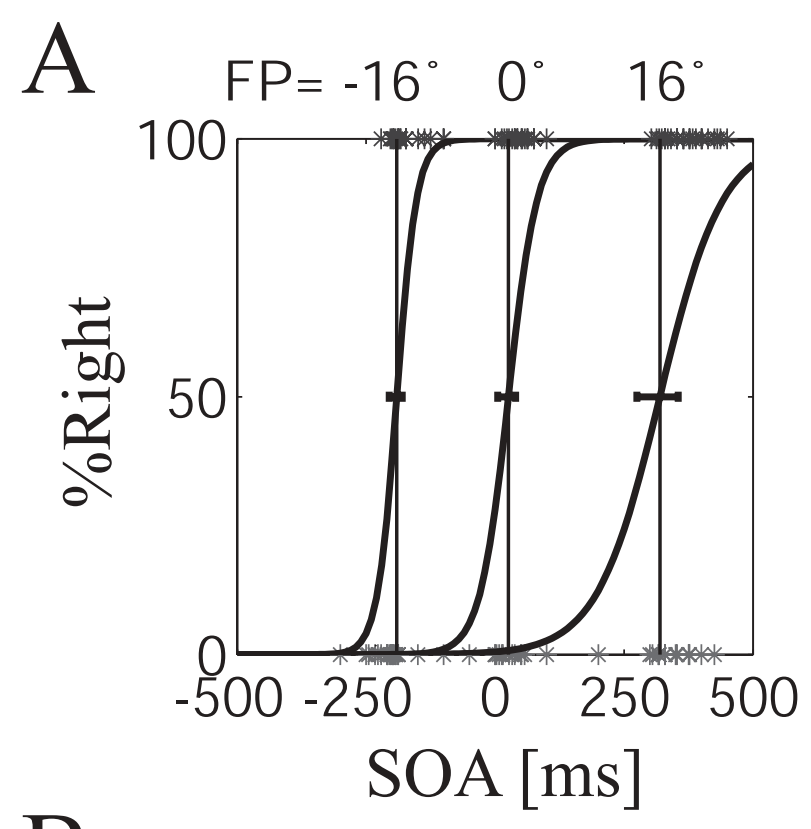

B

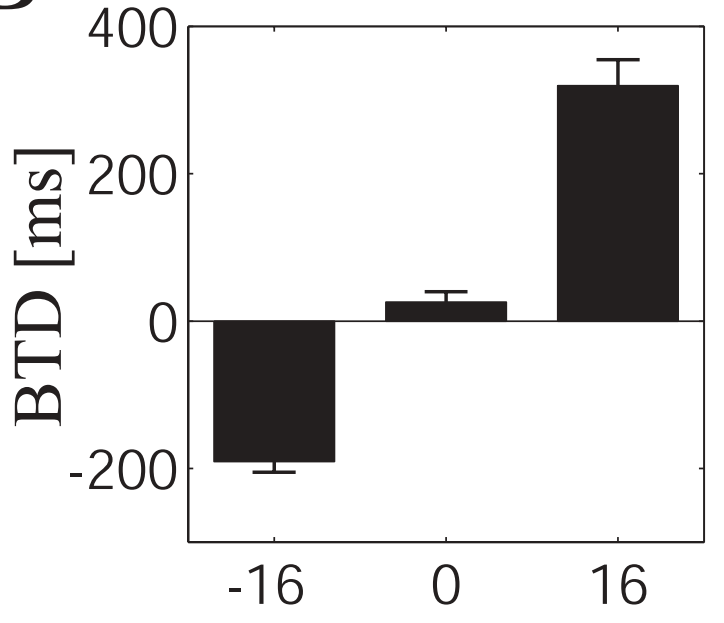

Fixation point [deg] 


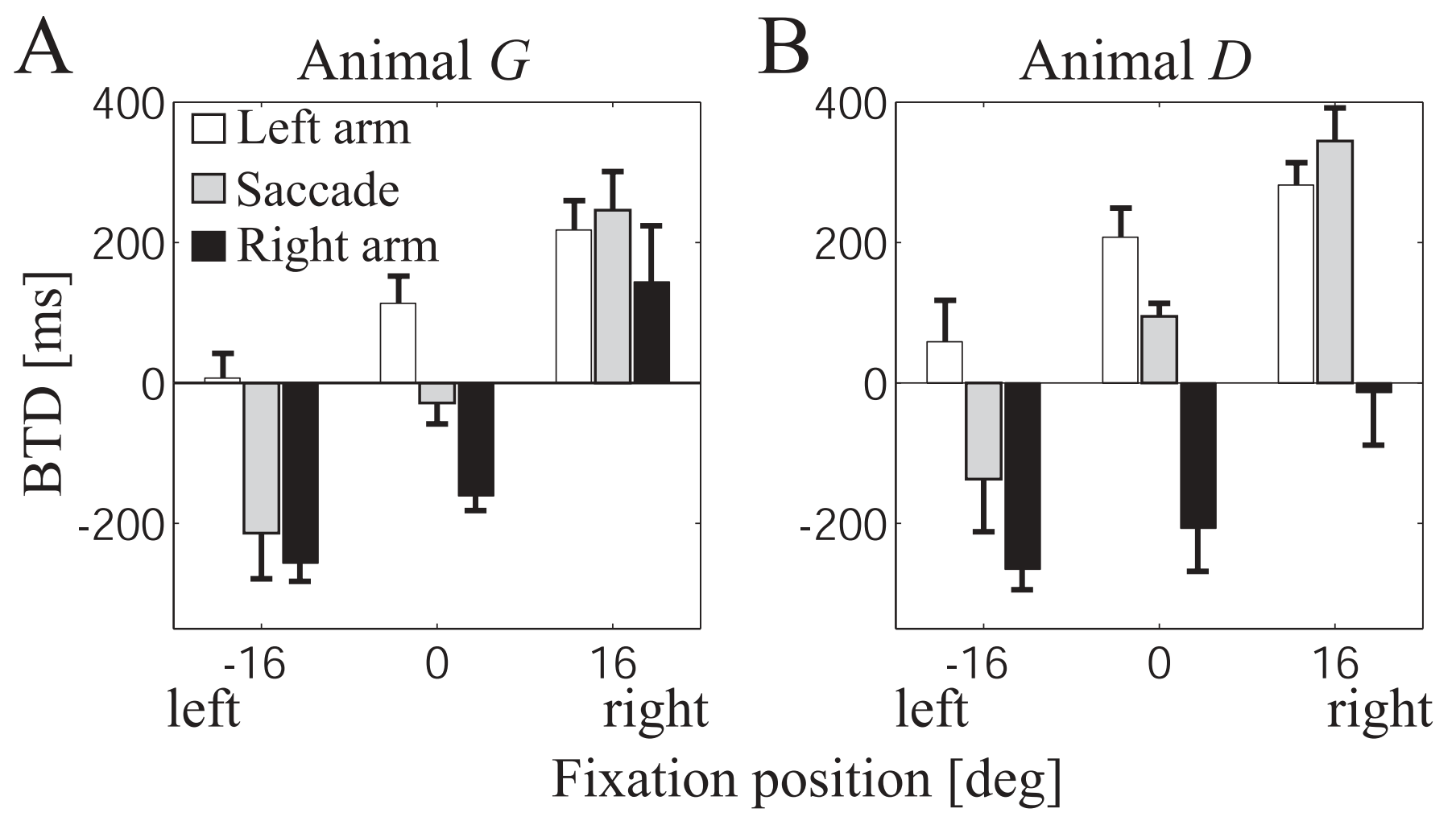

Scherberger et al, Figure 5 
A

B

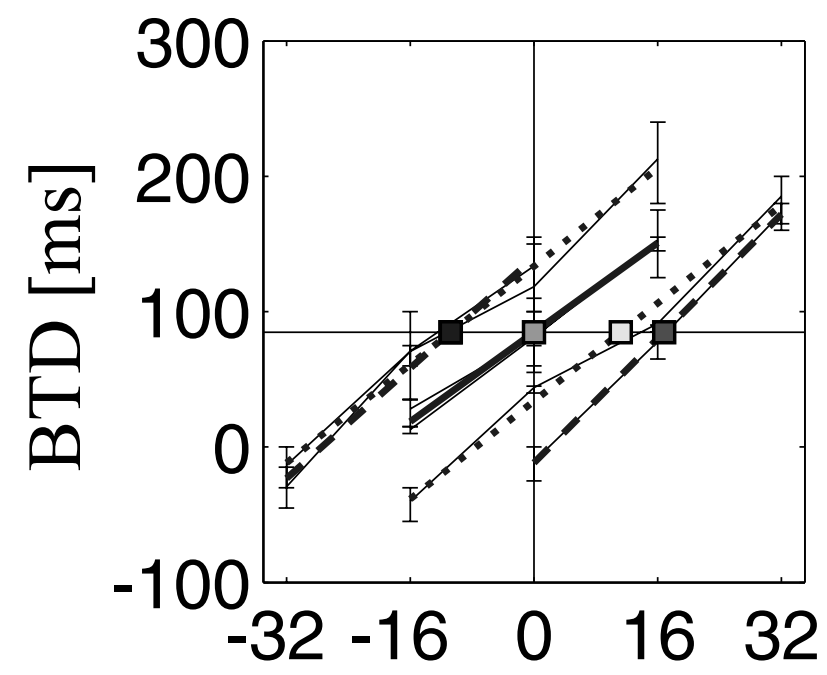

Fixation position [deg]

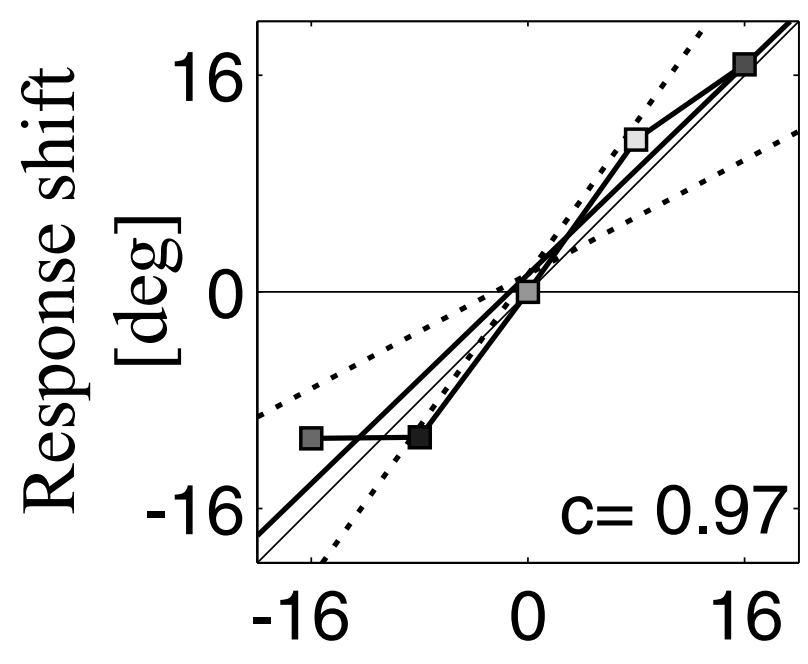

Head position [deg] 


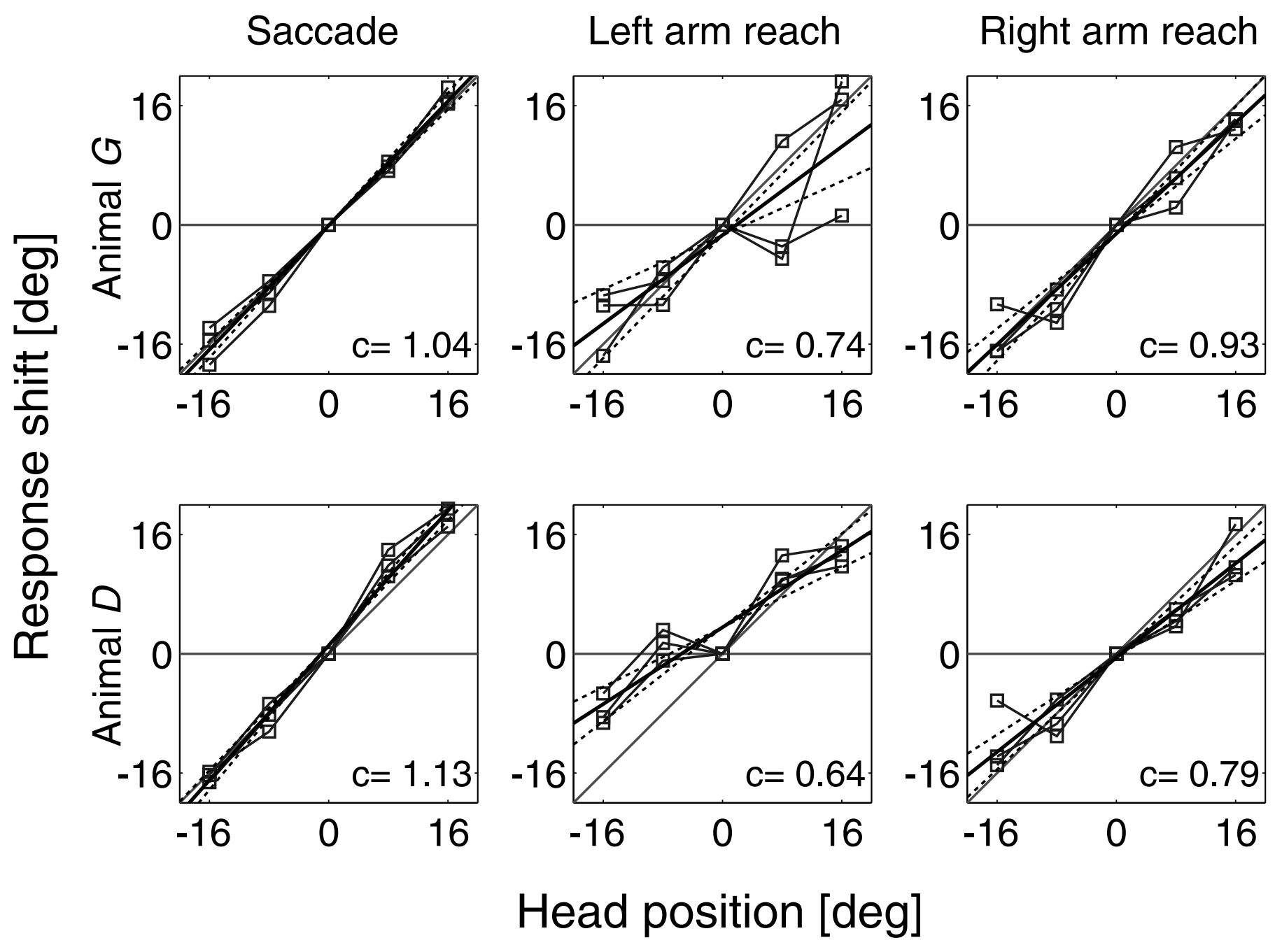

Scherberger et al, Figure 7 
A

B
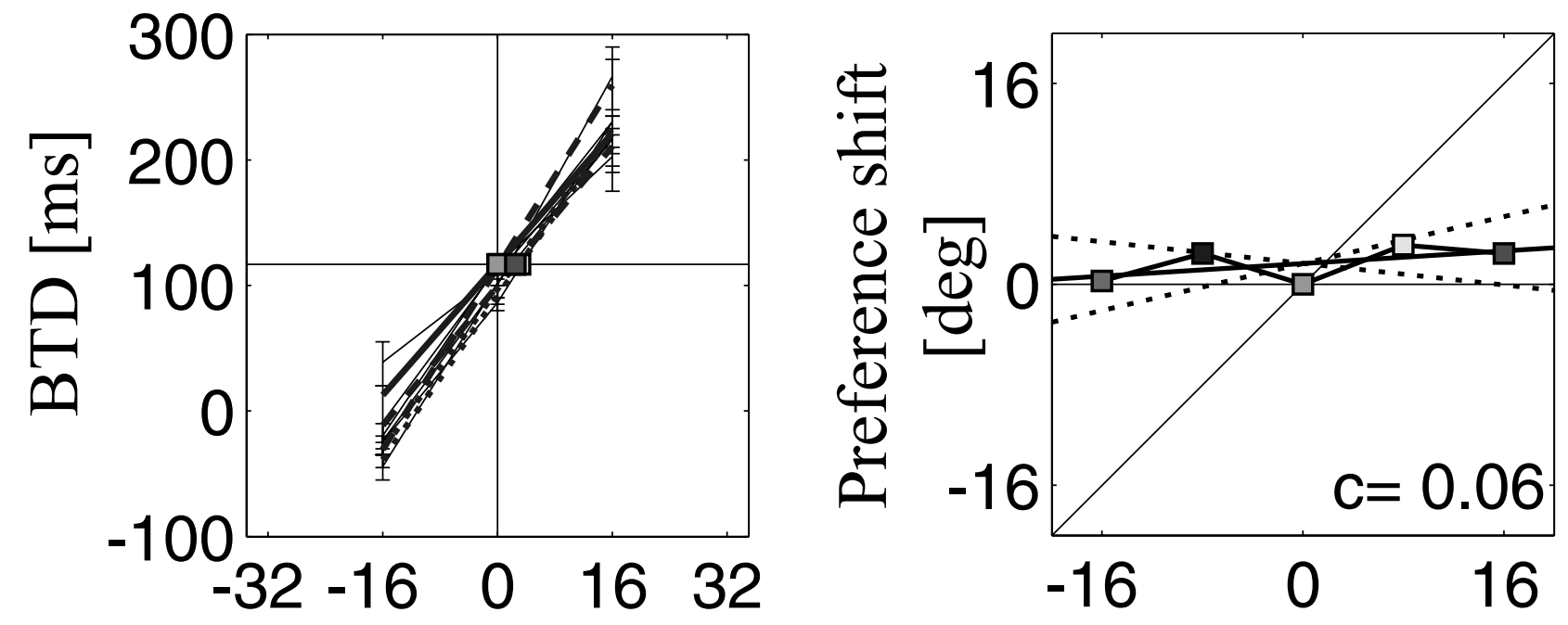

Fixation position [deg]

Trunk position [deg] 


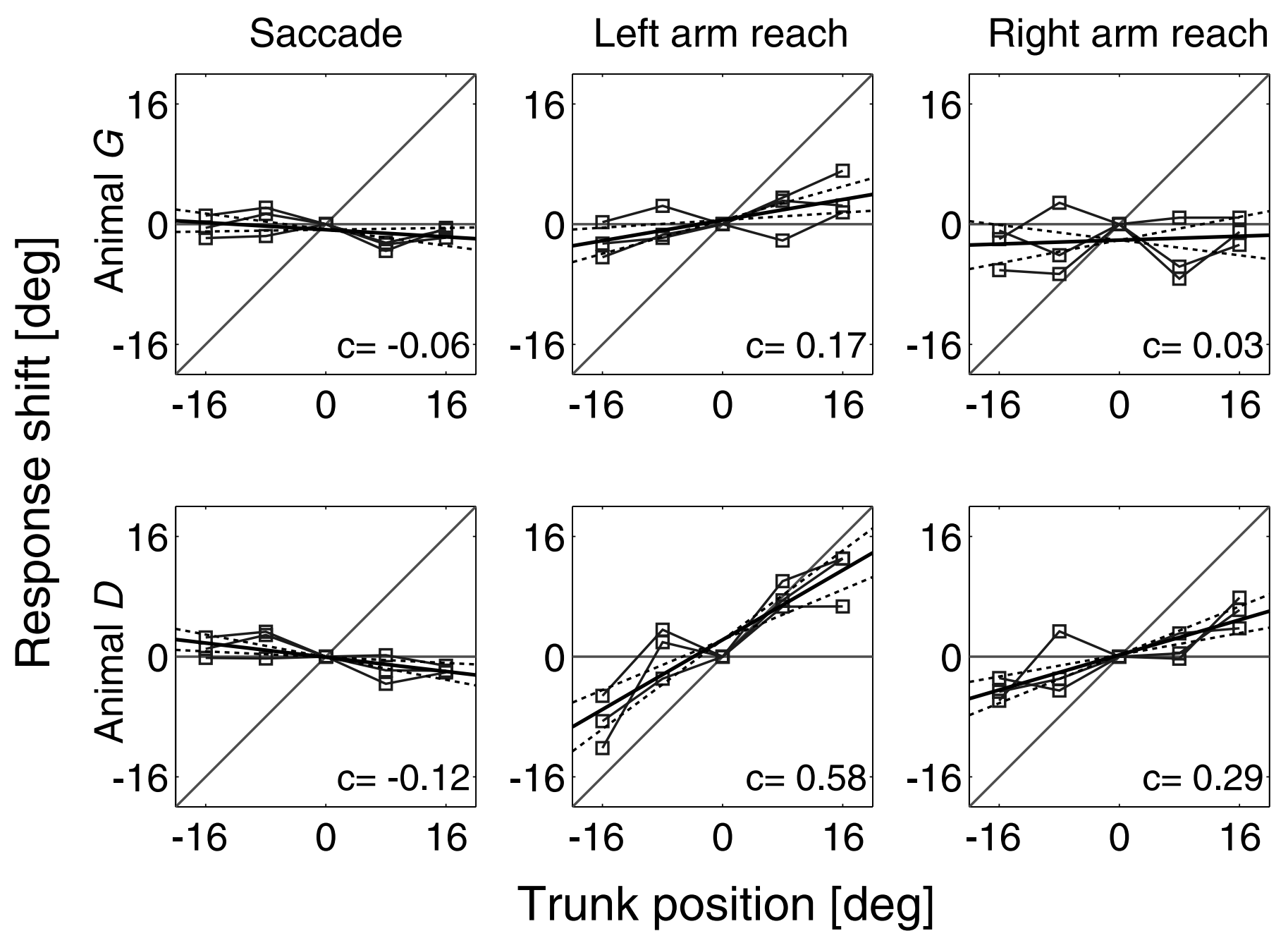

Scherberger et al, Figure 9 


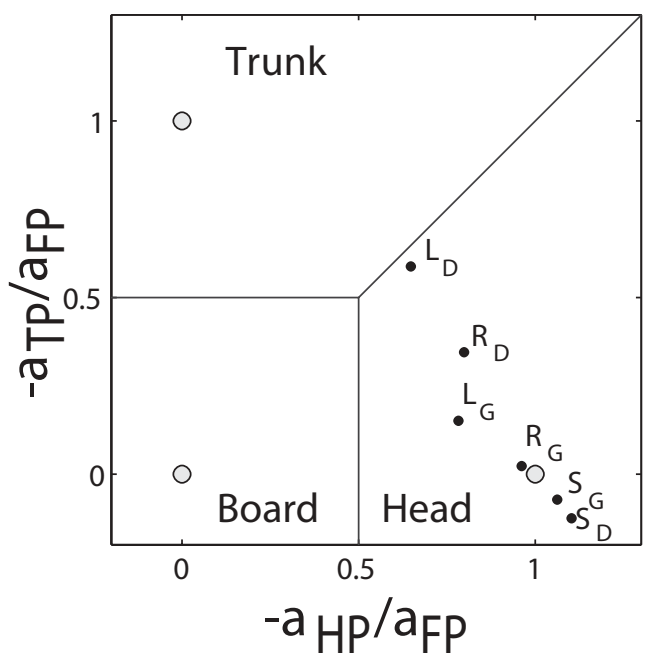

Scherberger et al, Figure 10 

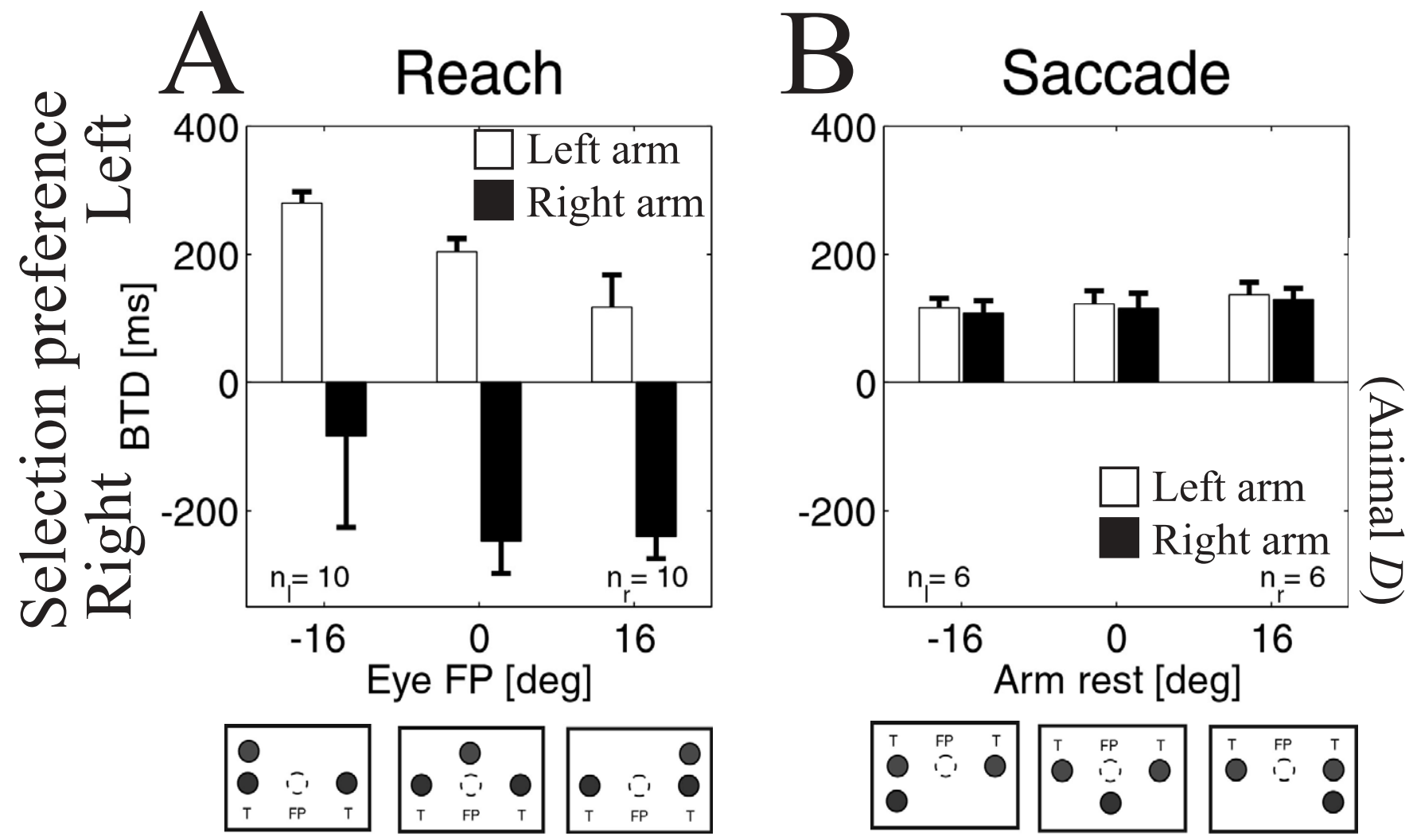

\begin{tabular}{|c|c|c|}
\hline $\begin{array}{lll} & F P & T \\
O & \ddots & O \\
0 & & \end{array}$ & $\begin{array}{|lll|}T & F P & T \\
O & -G & O \\
& -6 & \\
\end{array}$ & \begin{tabular}{llll|}
$T$ & $F P$ & $T$ \\
$O$ & $\vdots$ & $O$ \\
\end{tabular} \\
\hline
\end{tabular}


Animal Left arm reach
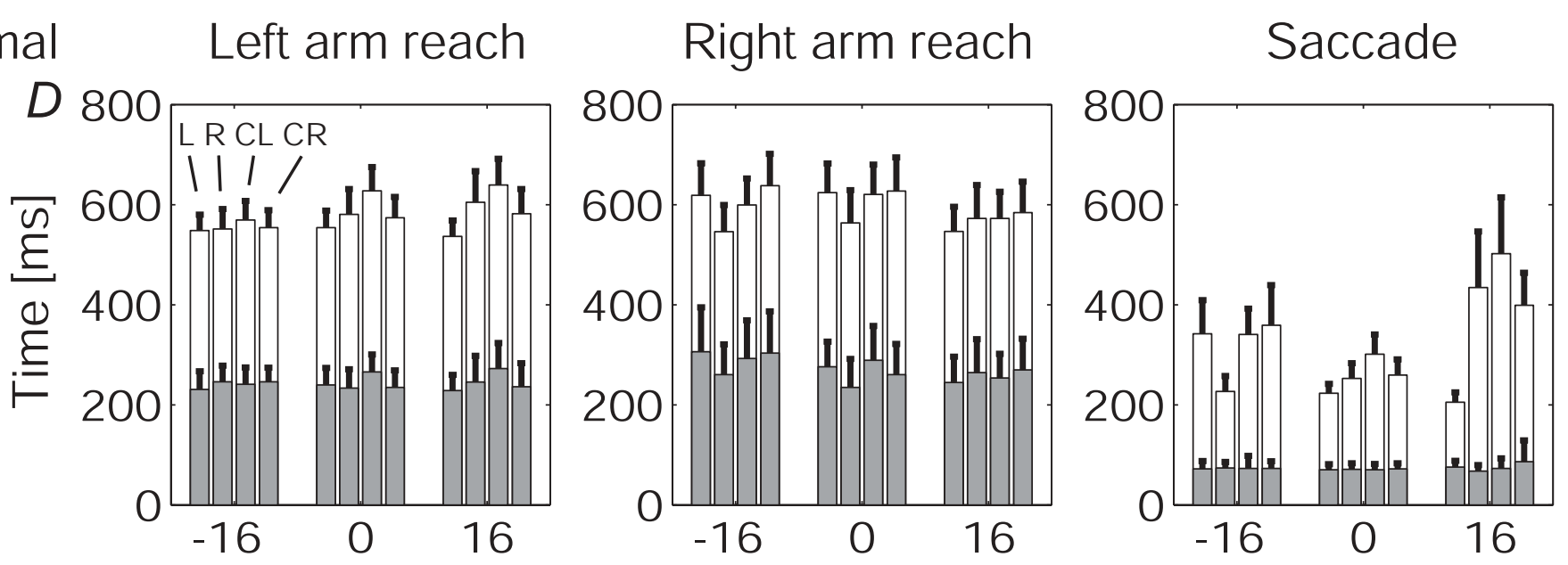

Animal
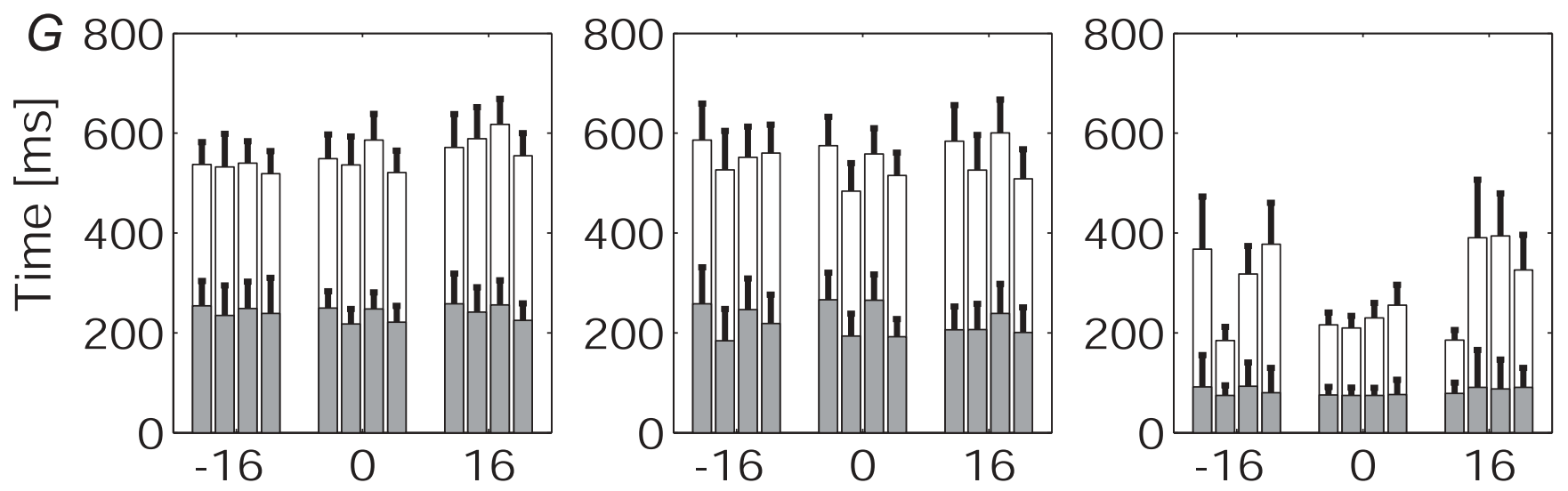

Fixation position [deg] 